\title{
Leçons tirées de la gestion de la syphilis au Nunavut, Canada, 2012 à 2020
}

\author{
Ameeta E Singh ${ }^{1 *}$, Kethika Kulleperuma ${ }^{2}$, Jenny Begin ${ }^{2}$, Jessica DeGuzman ${ }^{3}$, Diane Sammurtok ${ }^{4}$, \\ Obed Anoee ${ }^{4}$, Theresa Koonoo ${ }^{2}$, Jasmine Pawa ${ }^{2,5}$
}

\section{Résumé}

Contexte : Le Nunavut, territoire qui fait partie de I'Inuit Nunangat, est une vaste région dans le nord du Canada qui abrite une population de plus de 38000 personnes. La majorité de la population ( $85 \%$ ) s'identifie comme Inuit. Le Nunavut a connu une hausse importante du nombre de cas de syphilis infectieuse dans sa population hétérosexuelle depuis 2012. La gestion des maladies transmissibles, notamment la syphilis, est difficile en raison du roulement élevé du personnel et des longs délais de transport des spécimens. Les déterminants sociaux de la santé sont également des facteurs contributifs importants. Cette étude vise à décrire l'épidémiologie et les éléments du programme sur la syphilis infectieuse de 2012 à 2020 dans le but de mettre en évidence des interventions qui pourraient s'avérer bénéfiques.

Méthodes : La syphilis est une maladie à déclaration obligatoire au Nunavut, et tous les cas sont signalés au ministère de la Santé du territoire. Les cas ont été déclarés par un médecin consultant. Les données ont été analysées et publiées dans des rapports publics dans le cadre du programme de santé publique.

Résultats : De 2012 à 2020, 655 cas de syphilis infectieuse ont été signalés, dont $53 \%$ chez des femmes. Les taux d'infection étaient les plus élevés chez les personnes âgées de 20 à 39 ans. Les cas déclarés au cours de cette période variaient considérablement selon la région géographique, et la majorité des cas infectieux ont été signalés dans la région de Kivalliq. Malgré 48 cas signalés pendant la grossesse, il n'y a eu aucun cas confirmé de syphilis congénitale. Le personnel du programme a cerné les forces des interventions ainsi que les besoins continus, comme les ressources en langage clair disponibles dans plusieurs langues.

Conclusion : Malgré les défis logistiques liés à la gestion de la syphilis dans le territoire, les résultats globaux ont été positifs, et aucun cas congénital confirmé n'a été identifié. Nous attribuons cela à un effort coordonné de multiples partenaires, notamment des interventions clés d'infirmières de la santé publique et de représentants de la santé communautaire.
Cette oeuvre est mise à la disposition selon les termes de la licence internationale Creative Commons Attribution 4.0

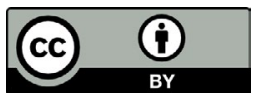

Affiliations

1 Division des maladies infectieuses, Université de l'Alberta, Edmonton, $A B$

${ }^{2}$ Gouvernement du Nunavut, ministère de la Santé, Iqaluit, NU

${ }^{3}$ Gouvernement du Nunavut, ministère de la Santé, Rankin Inlet, NU

${ }^{4}$ Gouvernement du Nunavut, ministère de la Santé, Arviat, NU

${ }^{5}$ Dalla Lana School of Public Health, Université de Toronto, Toronto, ON

\section{*Correspondance :} ameeta@ualberta.ca

Citation proposée : Singh AE, Kulleperuma K, Begin J, Deguzman J, Sammurtok D, Anoee O, Koonoo T, Pawa J. Leçons tirées de la gestion de la syphilis au Nunavut, Canada, 2012 à 2020. Relevé des maladies transmissibles au Canada 2022;48(2/3):115-24. https://doi.org/10.14745/ccdr.v48i23a08f

Mots-clés : syphilis, Canada, région de l'Arctique, épidémiologie, santé publique, représentant en santé communautaire

\section{Introduction}

Le Nunavut est un vaste territoire dans le nord du Canada qui fait partie de I'Inuit Nunangat et qui abrite plus de 38000 habitants, dont $85 \%$ s'identifient comme Inuits (1). Au cours des dernières années, le Nunavut a connu une augmentation importante du nombre de cas de syphilis infectieuse dans la population hétérosexuelle. Avant 2012, 5 cas ou moins par année étaient recensés dans cette population; lors de la période de 2016 à
2018, plus de 100 cas par année ont été recensés, ce qui est beaucoup plus élevé que le taux national. En 2017, le ministère de la Santé a retenu les services d'un spécialiste des maladies infectieuses pour aider le territoire à répondre à l'éclosion. À la suite d'un examen de 2017 de la prévention et du contrôle de la syphilis dans le territoire, plusieurs changements ont été apportés au programme de la syphilis au Nunavut, notamment 
une révision des lignes directrices territoriales sur la syphilis, une formation supplémentaire pour le personnel des soins de santé et de la santé publique, l'amélioration des activités de prévention par des représentants en santé communautaire et la détection, la gestion et le suivi de tous les cas de syphilis par le médecin consultant. Étant donné que de nombreuses femmes enceintes sont transférées à l'extérieur du territoire (en particulier de la région de Kivalliq) pour leur accouchement, le médecin consultant s'est efforcé de préparer des lettres sommaires sur tous les cas séropositifs de syphilis afin de faciliter les soins dans la province d'accueil.

La gestion de la syphilis au Nunavut est particulièrement difficile pour plusieurs raisons, notamment la vaste région géographique où les cas nécessitant des soins complexes sont triés dans trois provinces différentes au Canada, les emplacements éloignés de nombreuses collectivités, ce qui entraîne de longs retards dans le transport des spécimens et un manque de dotation en personnel. Les déterminants sociaux de la santé sont pertinents non seulement pour la syphilis, mais aussi pour l'état de santé général des Inuits. Les travaux de I'Inuit Tapiriit Kanatami, l'Organisation nationale inuite du Canada, ont fait ressortir l'importance de l'autodétermination et de miser sur les forces de la culture et de la langue inuites (2). Malgré ces défis, grâce aux efforts des membres de la collectivité et du personnel de santé, certains des résultats indésirables signalés dans d'autres administrations n'ont pas été observés, comme des taux plus élevés de cas de syphilis congénitale.

Notre objectif était de décrire l'épidémiologie de la syphilis infectieuse au Nunavut et de décrire les facteurs qui ont pu contribuer aux résultats observés. Ce travail est effectué et publié dans le cadre de la pratique en santé publique, notamment la planification et l'évaluation des programmes, et non comme recherche officielle.

\section{Méthodes}

\section{Infrastructure et flux des soins}

Diverses ressources gouvernementales et non gouvernementales ainsi que des publications ont été consultées pour décrire le flux des services de santé et de santé publique au Nunavut et pour décrire les rôles et les responsabilités du personnel de la santé au Nunavut.

\section{Dépistage de la syphilis}

Comme dans d'autres administrations canadiennes, les personnes symptomatiques ont accès à des services de diagnostic, tandis que les personnes asymptomatiques peuvent passer des tests de syphilis dans le cadre des processus de dépistage ou de recherche de cas (par la recherche des contacts et la notification). La syphilis est une maladie à déclaration obligatoire au Nunavut, et tous les cas sont signalés au ministère de la Santé du territoire. Les tests sérologiques sont utilisés pour diagnostiquer la syphilis au Nunavut à l'aide d'un algorithme de séquence inversée. L'analyse initiale porte sur un dosage immunoenzymatique de la syphilis (dosage immunologique microparticulaire par chimiluminescence d'Architect Syphilis TP, Abbot Laboratories, Abbott Park, Illinois, États-Unis). Un dosage immunoenzymatique positif est suivi d'un dosage quantitatif $\mathrm{d}^{\prime}$ anticorps réaginiques (test RPR BD Macro-Vue ${ }^{T M}$ RPR, Becton Dickinson Microbiology Systems, Mississauga, Ontario, Canada) et lorsqu'un test immunoenzymatique de la syphilis est positif pour la première fois, le résultat est confirmé à l'aide d'un essai d'agglutination des particules de Treponema pallidum (TPPA; Serodia -TPPA, Fujirebio Diagnostics Inc., Seguin, Texas, États-Unis). La syphilis est traitée conformément aux lignes directrices du Nunavut sur le traitement des infections transmissibles sexuellement (3) et les résultats positifs sont signalés aux autorités locales, territoriales et nationales de santé publique conformément aux lignes directrices territoriales sur les maladies à déclaration obligatoire (3).

\section{Déclaration et classification des cas}

Tous les cas de syphilis au Nunavut sont déclarés par les fournisseurs de soins de santé au coordonnateur régional des maladies transmissibles et à l'équipe d'épidémiologie territoriale au moyen d'un formulaire normalisé de définition des cas. Les renseignements suivants sont régulièrement entrés dans une base de données de surveillance : nom, date de naissance, sexe, région géographique, numéro d'assurance-maladie, raison du test, origine ethnique, détails sur les antécédents de syphilis, état de grossesse, résultats des tests de laboratoire sur la syphilis, date du traitement et stade de la maladie. Les renseignements suivants sont recueillis régulièrement sur le formulaire de définition de cas, mais ils ne sont pas systématiquement entrés dans la base de données : présence ou absence de symptômes, facteurs de risque et renseignements sur le partenaire. Les formulaires remplis ont été envoyés à un médecin consultant au cours de la période d'étude et la détermination des stades a été effectuée à l'aide des définitions de cas du Nunavut (voir le tableau supplémentaire : Définition de cas de surveillance au Nunavut pour la syphilis) (3). La confirmation de stade du cas est ensuite retournée au coordonnateur régional des maladies transmissibles et à l'épidémiologiste du territoire, qui travaillent en étroite collaboration avec les spécialistes territoriaux des maladies transmissibles et les agents de santé publique, au besoin. La recherche des contacts et d'autres mesures préventives de santé publique sont prises conformément aux lignes directrices territoriales.

\section{Collecte et analyse des données}

Le nombre de cas nationaux et territoriaux de syphilis infectieuse est extrait de l'ensemble de données national sur les maladies à déclaration obligatoire et de la base de données de surveillance de la syphilis du territoire, respectivement. Les données nationales et régionales sur la population du Nunavut sont tirées de Statistique Canada (4) et du Bureau de la statistique du Nunavut, respectivement. Tous les taux sont normalisés en 
fonction de la population normalisée de 2011. Les données sont analysées au moyen de Microsoft Excel et diffusées dans des rapports publics dans le cadre du programme de santé publique.

\section{Résultats}

\section{Infrastructure de soins de santé et flux de services}

Diagnostic, prise en charge et prévention de la syphilis : Le tableau 1 résume les rôles et les responsabilités du personnel de la santé qui fournit des services cliniques et préventifs aux personnes potentiellement touchées ou infectées par la syphilis au Nunavut. Le système de soins de santé du Nunavut dépend largement de contrats de soins infirmiers à court terme et de médecins suppléants, dont beaucoup viennent de l'extérieur du territoire (5). Sur les 25 collectivités du territoire, trois (lqaluit, Cambridge Bay et Rankin Inlet) ont des médecins à temps plein, tandis que les petites collectivités sont habituellement desservies par des médecins qui sont en rotation (5). Dans la plupart des collectivités, 22 cliniques communautaires, composées principalement $d$ 'infirmières en santé communautaire (infirmières autorisées travaillant dans un champ de pratique élargi), offrent des soins primaires et des services de courte durée (5). Les professionnels suppléants restent généralement pour des périodes relativement courtes et le roulement des médecins et des infirmières est très élevé; le taux de vacance dans certaines régions peut même atteindre $71 \%$ (3). Dans le cadre de ce processus, les directives médicales des infirmières en santé communautaire et des infirmières de la santé publique ont été examinées afin de trouver des occasions de mieux répartir le travail.

Les représentants en santé communautaire sont des membres inestimables de l'équipe des services de santé (5). Ils possèdent une expertise et une expérience importantes pour comprendre les collectivités dans lesquelles ils travaillent, notamment la capacité de travailler et d'établir des liens avec des personnes en inuktitut. Un programme fédéral de représentants en santé communautaire a été mis en œuvre en 1962 par la Direction générale des services médicaux de Santé et Bienêtre social Canada (6). Au sein du ministère de la Santé du Nunavut, il y a actuellement 33 postes de représentants en santé communautaire qui sont disponibles, dont 27 à temps plein, et tous les titulaires sont des femmes, à l'exception de deux cas. Même si, au cours des dernières années, le rôle principal des représentants en santé communautaire a été de se concentrer sur l'éducation en matière de santé, la promotion et la prévention des infections, les rôles varient d'une collectivité à I'autre. Au Nunavut, des représentants en santé communautaire ont été mobilisés pour aider à lutter contre la syphilis depuis le début de l'éclosion. Parmi les activités particulières, mentionnons l'organisation de séances d'information dans les écoles secondaires, l'accès à des préservatifs et à de l'information sur
Tableau 1 : Rôles et responsabilités du personnel de la santé qui fournit des services de prévention et de soins aux personnes potentiellement touchées ou infectées par la syphilis ${ }^{\mathrm{a}}$

\begin{tabular}{|c|c|}
\hline $\begin{array}{c}\text { Type de } \\
\text { fournisseur }\end{array}$ & Rôles \\
\hline $\begin{array}{l}\text { Fournisseur } \\
\text { responsable (e.g. } \\
\text { infirmière autorisée, } \\
\text { médecin, } \\
\text { sage-femme) }\end{array}$ & $\begin{array}{l}\text { Évaluer le patient } \\
\text { Effectuer des tests } \\
\text { Fournir le traitement } \\
\text { Remplir le formulaire de rapport sur la syphilis } \\
\text { ou le formulaire de rapport sur la syphilis pour } \\
\text { les nourrissons et le formulaire d'enquête sur } \\
\text { les contacts pour les ITS et le soumettre au } \\
\text { CRMT } \\
\text { Aviser, évaluer et traiter les contacts }\end{array}$ \\
\hline $\begin{array}{l}\text { Infirmière de santé } \\
\text { publique }\end{array}$ & $\begin{array}{l}\text { Évaluer le patient } \\
\text { Effectuer des tests } \\
\text { Organiser le traitement } \\
\text { Coordonner la notification des partenaires }\end{array}$ \\
\hline $\begin{array}{l}\text { Infirmière régionale } \\
\text { en contrôle } \\
\text { des maladies } \\
\text { transmissibles }\end{array}$ & $\begin{array}{l}\text { Réception et surveillance des résultats positifs } \\
\text { du test et de la sérologie de suivi pour la } \\
\text { syphilis } \\
\text { Réception des formulaires d'avis de syphilis du } \\
\text { fournisseur responsable } \\
\text { Veiller à ce que le traitement et le suivi de } \\
\text { la syphilis soient effectués par le fournisseur } \\
\text { responsable } \\
\text { Coordonner le transfert de l'information et des } \\
\text { recommandations médicales au fournisseur } \\
\text { responsable } \\
\text { Consulter un médecin consultant en ITS au } \\
\text { besoin pour la gestion des cas } \\
\text { Veiller à ce que la détermination du stade } \\
\text { de la syphilis soit effectuée par un médecin } \\
\text { consultant en ITS et soumise au STMT et à } \\
\text { l'épidémiologiste }\end{array}$ \\
\hline $\begin{array}{l}\text { Représentant en } \\
\text { santé communautaire }\end{array}$ & $\begin{array}{l}\text { Favoriser la santé et le bien-être dans la } \\
\text { collectivité en répondant aux besoins en } \\
\text { matière de santé publique et en appuyant } \\
\text { les priorités régionales et territoriales en } \\
\text { matière de santé publique en aidant les } \\
\text { personnes à acquérir des compétences et } \\
\text { des connaissances grâce à la prestation } \\
\text { d'information et à l'éducation en santé } \\
\text { publique }\end{array}$ \\
\hline $\begin{array}{l}\text { Consultant médical } \\
\text { territorial en ITS }\end{array}$ & $\begin{array}{l}\text { Fournir des conseils cliniques spécialisés et } \\
\text { du soutien au ministère de la Santé et aux } \\
\text { praticiens concernant la gestion des ITS, en } \\
\text { particulier la syphilis, notamment: } \\
\text { Détermination du stade, traitement et suivi } \\
\text { Soutien au programme territorial sur les } \\
\text { maladies transmissibles au sujet des ITS et } \\
\text { contrôle de l'éclosion de syphilis } \\
\text { Consultation sur les cas de syphilis difficiles ou } \\
\text { compliqués } \\
\text { Rédaction de lettres de consultation pour les } \\
\text { femmes enceintes chez qui on a diagnostiqué } \\
\text { la syphilis } \\
\text { Consultation et recommandations pour le suivi } \\
\text { des nourrissons nés de mères séropositives } \\
\text { pour la syphilis }\end{array}$ \\
\hline
\end{tabular}


Tableau 1 : Rôles et responsabilités du personnel de la santé qui fournit des services de prévention et de soins aux personnes potentiellement touchées ou infectées par la syphilis ${ }^{\text {a }}$ (suite)

\begin{tabular}{|l|l|}
\multicolumn{1}{c|}{$\begin{array}{c}\text { Type de } \\
\text { fournisseur }\end{array}$} & \multicolumn{1}{c|}{ Rôles } \\
\hline Épidémiologiste & $\begin{array}{l}\text { Analyser et interpréter les données sur la } \\
\text { syphilis } \\
\text { Rédiger des rapports }\end{array}$ \\
\hline $\begin{array}{l}\text { Spécialiste territorial } \\
\text { des maladies } \\
\text { transmissibles }\end{array}$ & $\begin{array}{l}\text { Coordonner le transfert de l'information à } \\
\text { l'extérieur de la province pour les cas et les } \\
\text { contacts } \\
\text { Collaborer avec les MH et les RSP sur les } \\
\text { protocoles territoriaux }\end{array}$ \\
\hline $\begin{array}{l}\text { Médecin hygiéniste/ } \\
\text { responsable de la } \\
\text { santé publique }\end{array}$ & $\begin{array}{l}\text { Fournir une expertise en santé publique pour } \\
\text { appuyer les opérations clés de protection de } \\
\text { la santé, de prévention des maladies et de } \\
\text { promotion de la santé }\end{array}$ \\
$\begin{array}{l}\text { Fournir un leadership et une expertise au } \\
\text { bureau de santé publique, y compris la } \\
\text { protection de la santé et le bureau de la santé } \\
\text { de la population } \\
\text { Établir et tenir à jour des normes et des } \\
\text { pratiques exemplaires en matière de santé } \\
\text { publique, et défendre la préservation et } \\
\text { l'amélioration de la santé des Nunavummiuts }\end{array}$ \\
\hline
\end{tabular}

Abréviations : CRMT, coordonnateur régional des maladies transmissibles; ITS, infection transmise sexuellement; $\mathrm{MH}$, médecin-hygiéniste; RSP, responsable de la santé publique; STMT, spécialiste territorial des maladies transmissibles

${ }^{a}$ Ce résumé porte sur les soins de la syphilis et sur le fait qu'il y a beaucoup de personnes qui travaillent dans le système de santé et qui ne sont pas reconnues ici. Cela comprend, sans s'y limiter, le personnel administratif, les programmes de voyage pour raisons médicales, et les membres des équipes des politiques, des finances, des communications et des ressources humaines

les options de contraception et l'élaboration de matériel de promotion de la santé. Parmi les autres activités, mentionnons la tenue de kiosques d'information sur la santé, l'aide aux services de traduction et le soutien à la navigation dans le système de santé. Dans certaines collectivités, la tenue d'émissions de radio mensuelles a facilité la diffusion d'information et a permis aux membres de la collectivité de téléphoner et de poser des questions.

Les personnes qui ont besoin de services médicaux supplémentaires sont habituellement transportées à l'extérieur du territoire, parfois à un autre endroit situé dans le territoire, comme le résume la figure 1.

Dépistage de la syphilis : Des spécimens de la région de Kitikmeot ont été soumis à DynaLife Laboratories (Edmonton, Alberta) et des régions de Qikiqtaaluk et Kivalliq au laboratoire de l'Hôpital général de Qikiqtani (lqaluit, Nunavut). Étant donné que le transport en provenance de collectivités éloignées n'a lieu que certains jours de la semaine, selon les horaires de vol, et qu'il peut être nécessaire de transférer des spécimens à un autre centre de tri situé dans le territoire, le délai nécessaire pour analyser les résultats peut être très long. Dans les petites collectivités, le temps nécessaire pour obtenir les résultats du test de syphilis varie souvent de 8 à 12 jours et peut aller jusqu'à quelques semaines. De plus, les femmes enceintes qui avaient
Figure 1 : Exemples de modes de déplacement des patients du Nunavut vers une destination à l'extérieur du territoire ${ }^{a}$

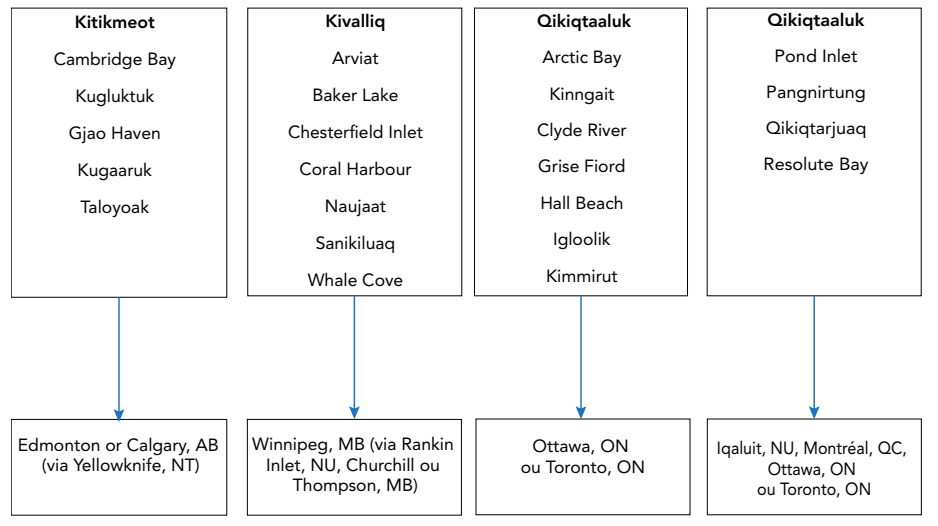

Abréviations : AB, Alberta; MN, Manitoba; NU, Nunavut; NT, Territoires du Nord-Ouest; ON, Ontario; QC, Québec

aeuillez consulter la référence (7)

Remarque : Ce qui précède n'est pas une liste exhaustive des collectivités du Nunavut. Il s'agit d'exemples de modes de déplacement. II est également important de noter que les noms des collectivités peuvent être désuets (e.g. Kinngait était auparavant connu sous le nom de Cape Dorset)

besoin d'être transportées à l'extérieur du territoire ont souvent été testées dans la province d'accueil ou testées de nouveau à leur arrivée, ce qui a entraîné une variabilité dans les résultats des tests, en particulier le test rapide de la réagine plasmique (RPR), en raison des différences entre les algorithmes d'essai et les types de tests utilisés.

\section{Épidémiologie}

Au total, 655 cas ont été signalés au Nunavut de 2012 à 2020, et $95 \%$ des cas déclarés concernaient des personnes d'origine inuite (un pourcentage semblable à celui de la population générale, avec $85 \%$ s'identifiant comme des Inuits). Les taux normalisés de syphilis infectieuse selon l'âge et le sexe au Nunavut et au Canada (figure 2) montrent des taux bien supérieurs aux taux nationaux pour les hommes et les femmes. Contrairement aux taux nationaux, où les taux normalisés selon l'âge étaient beaucoup plus élevés chez les hommes, les taux territoriaux normalisés selon l'âge étaient semblables chez les hommes et les femmes. La figure $\mathbf{3}$ montre les taux normalisés de syphilis infectieuse selon l'âge au Nunavut par région et année et met en évidence la propagation géographique et la croissance relative des cas dans le territoire et les trois régions. Étant donné qu'avant 2012, on signalait cinq cas par année ou moins, l'observation d'une augmentation des cas infectieux en 2012 (plus de 20 cas) a entraîné la déclaration d'une éclosion en 2012 et les premiers cas ont été signalés à lqaluit (figure 4). Au départ, l'éclosion était concentrée dans la région de Qikiqtaaluk, puis elle s'est déplacée dans la région de Kivalliq. Étant donné que de nouveaux cas infectieux continuent d'être déclarés, l'éclosion est actuellement considérée comme active. Qikiqtaaluk comptait plus de $50 \%$ de la population totale du Nunavut et signale des cas depuis 2012, avec des fluctuations notables au fil du temps. La croissance du nombre de cas dans la région de Kivalliq a considérablement augmenté en 2016, mais la tendance 
semble diminuer depuis. Dans l'ensemble, $53 \%(n=349)$ des cas ont été signalés chez les femmes, et le ratio global homme-femme au Nunavut était de 0,9 (fourchette de 0,7 à 1,0) (tableau 2). Dans l'ensemble, $54 \%$ des cas ont été classés au stade primaire $(n=356), 18 \%$ au stade secondaire $(n=117)$, $27 \%$ au stade de latence précoce $(n=175)$ et $1 \%$ à un stade inconnu $(n=7)$.

Figure 2 : Taux normalisés de syphilis infectieuse selon l'âge et le sexe au Nunavut et au Canada, de 2012 à 2020

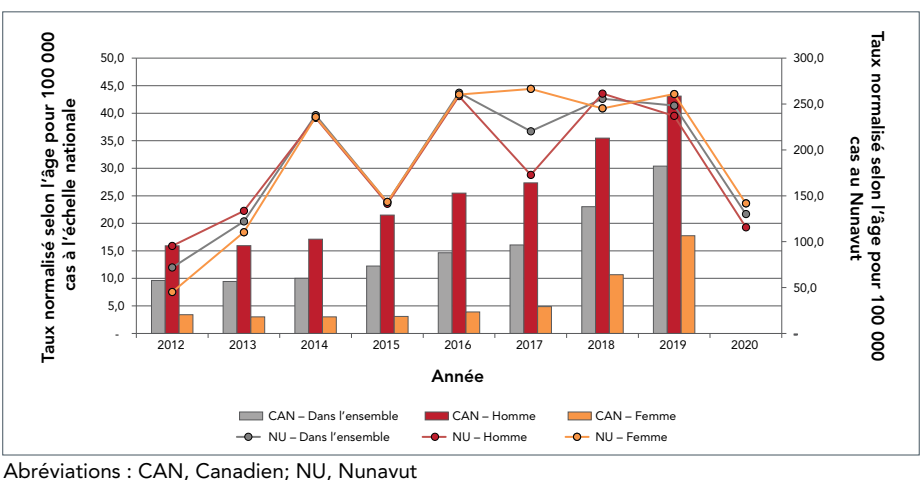

Figure 3 : Taux normalisés de syphilis infectieuse selon l'âge au Nunavut par région et année, de 2012 à 2020 $(n=655)$

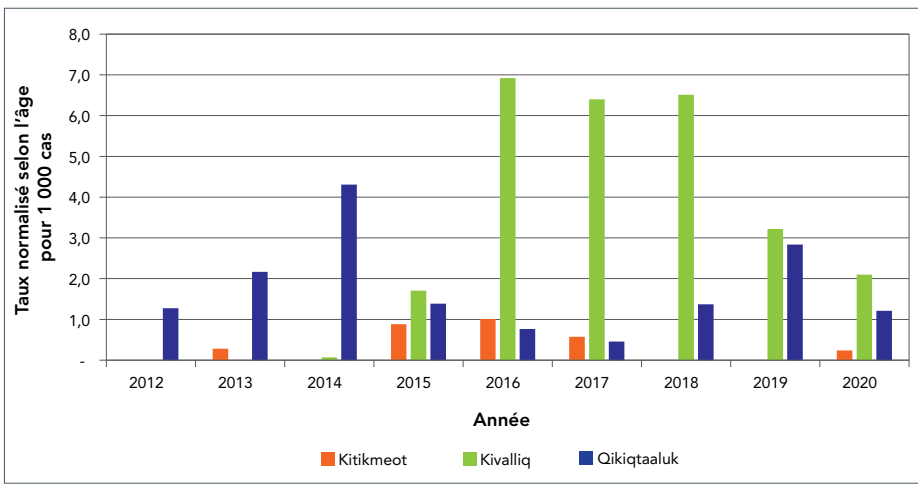

Figure 4 : Courbe épidémiologique de la syphilis infectieuse au Nunavut par région, de 2011 à 2020

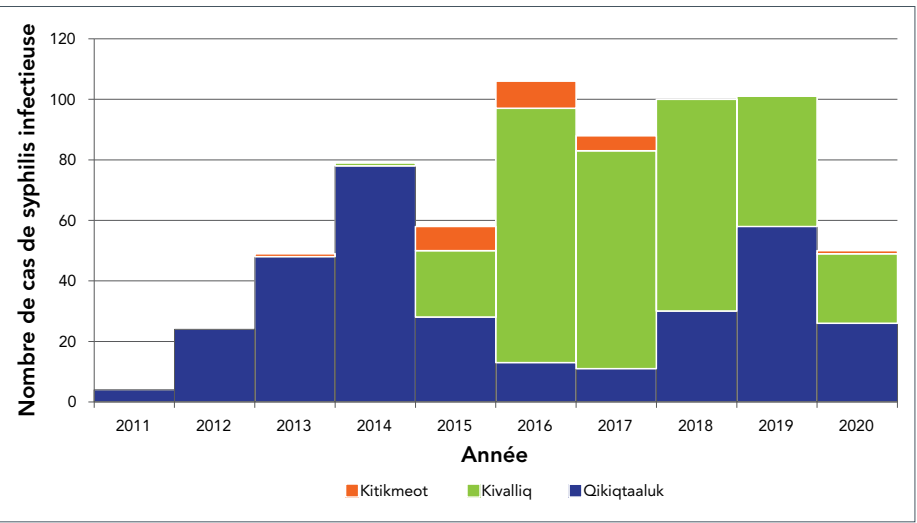

Tableau 2 : Cas de syphilis infectieuse au Nunavut selon I'année, le sexe, le ratio homme-femme, le groupe d'âge, le stade de la syphilis et la région, de 2012 à 2020

\begin{tabular}{|c|c|c|c|c|}
\hline \multirow{2}{*}{ Description } & \multicolumn{4}{|c|}{ Région } \\
\hline & Nunavut & Kitikmeot & Kivalliq & Qikiqtaaluk \\
\hline $\begin{array}{l}\text { Nombre total } \\
\text { de cas }\end{array}$ & 655 & 24 & 315 & 316 \\
\hline 2012 & 24 & 0 & 0 & 24 \\
\hline 2013 & 49 & $<5$ & $<5$ & 48 \\
\hline 2014 & 79 & $<5$ & $<5$ & 78 \\
\hline 2015 & 58 & 8 & 22 & 28 \\
\hline 2016 & 106 & 9 & 84 & 13 \\
\hline 2017 & 88 & 5 & 72 & 11 \\
\hline 2018 & 100 & 0 & 70 & 30 \\
\hline 2019 & 101 & 0 & 43 & 58 \\
\hline 2020 & 50 & $<5$ & $20+$ & 26 \\
\hline \multicolumn{5}{|l|}{ Sexe } \\
\hline Femme & 349 (53 \%) & 14 & 176 & 159 \\
\hline Homme & 306 (47 \%) & 10 & 139 & 157 \\
\hline $\begin{array}{l}\text { Ratio } \\
\text { homme-femme }\end{array}$ & 0,9 & 0,7 & 0,8 & 1,0 \\
\hline 2012 & 1,7 & 0 & 0 & 0,6 \\
\hline 2013 & 1,2 & $<5$ & $<5$ & 0,8 \\
\hline 2014 & 0,8 & $<5$ & $<5$ & 1,2 \\
\hline 2015 & 0,9 & 1,7 & 0,8 & 1,2 \\
\hline 2016 & 0,9 & 0,8 & 1,4 & 0,4 \\
\hline 2017 & 0,6 & 4,0 & 1,8 & 0,8 \\
\hline 2018 & 1,0 & 0 & 1,1 & 0,9 \\
\hline 2019 & 0,9 & 0 & 0,8 & 1,3 \\
\hline 2020 & 0,7 & $<5$ & 1,9 & 1,4 \\
\hline \multicolumn{5}{|l|}{ Groupe d'âge } \\
\hline $\begin{array}{l}\text { Moins de } \\
15 \text { ans }\end{array}$ & $5(1 \%)$ & $<5$ & $<5$ & $<5$ \\
\hline De 15 à 19 ans & 92 (17\%) & 7 & 65 & 38 \\
\hline De 20 à 24 ans & 110 (22 \%) & 5 & 89 & 49 \\
\hline De 25 à 29 ans & 143 (22 \%) & 9 & 59 & 79 \\
\hline De 30 à 39 ans & 147 (24 \%) & $<5$ & 64 & 93 \\
\hline 40 ans et plus & 158 (14 \%) & $<5$ & 35 & 55 \\
\hline \multicolumn{5}{|l|}{ Stade } \\
\hline Primaire & 356 (54 \%) & 13 & 178 & 165 \\
\hline Secondaire & 117 (18\%) & $<5$ & 49 & 64 \\
\hline $\begin{array}{l}\text { Latence } \\
\text { précoce }\end{array}$ & 175 (27 \%) & 7 & 88 & 80 \\
\hline $\begin{array}{l}\text { Erreur de } \\
\text { documentation }\end{array}$ & $7(1 \%)$ & 0 & 0 & 0 \\
\hline
\end{tabular}


Les cas ont été répartis entre tous les groupes d'âge, la majorité des cas ayant entre 20 et 39 ans. Au cours de cette période, 48 femmes enceintes ont reçu un diagnostic de syphilis infectieuse au Nunavut, avec plus de $66 \%$ de cas dans la région de Kivalliq, plus de $30 \%$ dans la région de Qikiqtaaluk et moins de $5 \%$ dans la région de Kitikmeot (les chiffres exacts ne sont pas disponibles en raison du faible nombre de cas déclarés). Toutes les femmes enceintes ont été traitées conformément aux lignes directrices du Nunavut (3). En ce qui concerne les résultats néonatals, 40 nourrissons ont été classés comme des non-cas (transfert d'anticorps maternels uniquement), 4 étaient des avortements thérapeutiques ou spontanés ou des mortinaissances (non dues à la syphilis), 3 étaient des cas de syphilis congénitale probables et 1 cas était en attente d'information de suivi au moment de la rédaction. Parmi les cas de transfert maternel, 23 (57\%) des femmes enceintes ont été traitées avant 20 semaines de gestation et 4 (10\%) après 30 semaines de gestation.

\section{Discussion}

Après avoir observé peu ou pas de transmission endémique de la syphilis, le Nunavut a connu une hausse importante du nombre de cas de syphilis infectieuse depuis 2012. Le nombre et les taux ont culminé en 2016 et sont demeurés stables chaque année jusqu'en 2019. Cette augmentation a été suivie d'une baisse en 2020. La raison de la baisse observée en 2020 n'était pas claire, car les données sur les tests de dépistage de la syphilis n'étaient pas disponibles pour la période observée. Il est possible que les chiffres et le taux aient diminué en raison de la réduction des interactions avec le système de santé dans le territoire en raison de la pandémie de maladie à coronavirus de 2019 (COVID-19). Des tendances semblables ont été signalées dans d'autres administrations (8). Une autre explication possible est que les mesures de prévention mises en œuvre pendant la pandémie de COVID-19, comme les recommandations de rester à la maison et la fermeture d'entreprises non essentielles, pourraient avoir entraîné un changement de comportement sexuel lié à la distanciation physique et, par conséquent, une diminution réelle du nombre de cas (8).

L'éclosion de syphilis infectieuse a principalement été transmise dans la population hétérosexuelle, contrairement au taux canadien global, qui est plus élevé chez les hommes. Par conséquent, 48 femmes enceintes ont reçu un diagnostic de syphilis infectieuse au cours de la période à l'étude, dont environ les deux tiers provenaient de la région de Kivalliq. Les femmes de la région de Kivalliq qui avaient une syphilis infectieuse pendant leur grossesse se rendaient habituellement à Winnipeg par avion entre la $34^{\mathrm{e}}$ et la $36^{\mathrm{e}}$ semaine de gestation et y restaient jusqu'à l'accouchement. La plupart des femmes enceintes de la région de Kivalliq provenaient de très petites collectivités (moins de 2000 personnes) qui ont souvent un petit centre de santé communautaire offrant des niveaux variables de soutien et de services du personnel médical. Le centre principal, Rankin Inlet, dispose d'un centre de naissance avec des sages-femmes qui offrent du soutien pour les accouchements sans complications, mais comme les nourrissons nés de mères traitées pour la syphilis infectieuse pendant la grossesse doivent être évalués pour la syphilis congénitale par des pédiatres expérimentés, toutes ces femmes ont été transférées à Winnipeg pour l'accouchement. De plus, le centre de naissance de Rankin Inlet est actuellement fermé pour des raisons de dotation. Les femmes qui ont reçu un diagnostic de syphilis infectieuse après 20 semaines de gestation ont été transférées à Winnipeg aux fins de surveillance, car le traitement peut entraîner une réaction de Jarisch Herxheimer, soit le déclenchement prématuré du travail (9). Les femmes enceintes de la région de Qikiqtaaluk sont régulièrement transférées à l'hôpital général de Qikiqtani pour y être traitées (après plus de 20 semaines de gestation) et y accoucher. Fait intéressant, deux séries rétrospectives récentes de cas de femmes enceintes diagnostiquées avec la syphilis et traitées à Winnipeg et en Alberta n'ont signalé aucun événement maternel ou fœtal grave après le traitement $(10,11)$. Les déplacements pour raisons médicales au Nunavut représentent $20 \%$ des dépenses de santé au Nunavut en raison de l'absence relative de routes et de la dépendance au transport aérien (7); cela est pertinent en ce qui concerne les femmes enceintes à plus de 20 semaines de gestation avec syphilis infectieuse qui sont régulièrement transférées dans un grand centre de traitement. Les études de Winnipeg et de l'Alberta, bien que modestes, n'appuient pas la pratique courante $d^{\prime}$ 'admission à l'hôpital pour le traitement de la syphilis infectieuse à la fin de la grossesse. Toutefois, étant donné la nature rétrospective restreinte des études, il se peut que ces études n'aient pas identifié un sous-ensemble de femmes qui pourraient être à risque d'événements indésirables graves.

En ce qui concerne les résultats néonatals de la syphilis infectieuse pendant la grossesse, nous n'avons trouvé aucun cas confirmé de syphilis congénitale. La transmission verticale de la syphilis se produit à tous les stades de la syphilis et à chaque trimestre de la grossesse, mais le risque de transmission augmente avec le stade précoce de la syphilis et l'âge gestationnel ultérieur (9). L'infection fœtale survient dans plus de $50 \%$ des cas de syphilis précoce non traitée (9). Le faible taux de résultats néonatals indésirables observé au Nunavut peut être attribué à de multiples facteurs, notamment les tests de routine de syphilis chez toutes les femmes enceintes ou pour la majorité d'entre elles au début de la grossesse et le traitement rapide en cas de diagnostic de la syphilis. La majorité (ou peut-être la totalité) des femmes se soumettent à des tests de dépistage de la syphilis, souvent au début de la grossesse en raison de la petite taille des collectivités. De plus les femmes ont généralement accès à des services, car elles doivent habituellement être transférées de leur collectivité pour l'accouchement. Toutes les études ont démontré que les taux les plus faibles de syphilis congénitale et d'issues indésirables de la grossesse découlent d'un traitement maternel précoce et 
adéquat au début de la grossesse $(12,13)$. De plus, les lignes directrices sur la syphilis au Nunavut recommandent le dépistage systématique de la syphilis infectieuse à la période de gestation de 24 à 28 semaines, ce qui permet de déterminer et de traiter plus tôt la réinfection. Afin de minimiser l'impact de la variation des résultats des tests RPR en laboratoire, tous les cas de syphilis infectieuse revenant de l'extérieur du territoire passent un nouveau test RPR à leur retour afin que ce résultat puisse être utilisé pour éclairer le suivi.

Tous les membres de l'équipe des services de santé jouent un rôle important dans les soins et la prévention des personnes atteintes de syphilis. Bien que les fournisseurs de première ligne (comme les infirmières en santé communautaire) jouent un rôle essentiel dans l'évaluation initiale et la coordination du traitement, le coordonnateur régional des maladies transmissibles est essentiel pour s'assurer que le traitement et le suivi sérologique ont lieu, que les partenaires sont contactés et qu'on leur offre des tests et des traitements. Le coordonnateur régional des maladies transmissibles, en consultation avec le médecin consultant territorial responsable du traitement de la syphilis, veille également à ce que les renseignements médicaux pertinents soient transmis à d'autres fournisseurs de soins qui peuvent participer aux soins du patient. Le consultant spécialiste territorial des maladies transmissibles (qui est souvent aussi infirmier ou infirmière) s'assure que les renseignements pertinents sont transmis aux clients pour les soins et le suivi à l'extérieur du territoire, et il travaille avec le médecin hygiéniste ou le responsable de santé publique pour le suivi des protocoles territoriaux. Le rôle des épidémiologistes territoriaux est de fournir des données épidémiologiques exactes et opportunes, ce qui est essentiel à la planification des programmes. Au fil des ans, le Nunavut a connu un taux de roulement élevé du personnel permanent et temporaire de remplacement des services de santé, ce qui a été particulièrement difficile pendant l'éclosion de syphilis et la pandémie de COVID-19. La mise à jour des lignes directrices, des algorithmes et des protocoles d'une page sur la syphilis au Nunavut a facilité ces transitions.

II ne faut pas sous-estimer le rôle des représentants en santé communautaire. Voici une citation de la Commission royale sur les peuples autochtones : [Traduction] «L'un des programmes les plus efficaces en matière de promotion de la santé des Autochtones est le Programme des représentants en santé communautaire » (14). Dans de nombreuses collectivités nordiques et isolées, y compris au Nunavut, le roulement des professionnels de la santé est extrêmement élevé; le représentant en santé communautaire est le seul à assurer la continuité des soins dans certaines collectivités. «Les représentants en santé communautaire sont les personnes en qui les membres de la collectivité ont confiance et auxquelles ils ont affaire en ce qui concerne l'information et les services en matière de santé. " (14). Il y a peu de données au Canada sur l'incidence du rôle des représentants en santé communautaire dans la prévention et le contrôle des maladies transmissibles, y compris la syphilis. L'analogie la plus proche dans la documentation publiée est peut-être l'impact du rôle des pairs travailleurs. Un examen systématique a révélé que les interventions d'éducation par les pairs sont associées à une multiplication par trois des tests de dépistage du $\mathrm{VIH}$, avec un impact constant sur le changement de comportement pendant plus de 24 mois (15). Les pairs fournissent un soutien informationnel, émotionnel et d'affiliation pour l'utilisation des services de dépistage (16).

Les discussions sur les maladies transmissibles en particulier et l'état de santé en général doivent être considérées dans le contexte des déterminants sociaux de la santé. L'Inuit Tapiriit Kanatami a créé des ressources axées sur les Inuits pour informer les organisations et le gouvernement, notamment la pratique en santé publique et les principaux déterminants sociaux de la santé des Inuits canadiens (2). Lorsqu'ils discutent des défis en matière de santé auxquels sont confrontés les Inuits, les chercheurs et d'autres intervenants doivent tenir compte de la force et de la richesse des connaissances qui caractérisent la culture inuite. Les répercussions du colonialisme, y compris les programmes des pensionnats, ont été vastes et profondes (17). La première école gouvernementale pour les Inuits a ouvert ses portes à Chesterfield Inlet en 1951. On estime que $75 \%$ des enfants et des jeunes Inuits âgés de 6 à 15 ans étaient inscrits dans ces écoles en juin 1964 et qu'au moins 3000 Inuits qui ont fréquenté les pensionnats sont encore en vie aujourd'hui. L'occidentalisation et la colonisation ont été identifiées dans la littérature comme des influences négatives sur la santé sexuelle en raison de la perte de la sagesse et des connaissances accumulées par les Inuits au sujet du cycle de vie, de la santé génésique et de la planification familiale ainsi que des modes de vie traditionnels qui intègrent à la fois des compétences pratiques et des principes culturels $(18,19)$. Ces facteurs ont contribué à perturber considérablement les structures et les relations familiales. Les abus sexuels, y compris envers les enfants, sont une préoccupation importante. Des organisations comme Pauktuutit ont mis au point des ressources pour aider les personnes et les organisations à régler ce problème (20). Dans un récent sondage mené auprès de jeunes Inuits, la plupart d'entre eux ont déclaré ne pas avoir utilisé Internet pour obtenir des renseignements sur la santé sexuelle (18). Pour citer l'un des représentants en santé communautaire, les médias sociaux envoient souvent un "mauvais message " au sujet des relations. Dans le même sondage, les parents ou les fournisseurs de soins ont été déclarés comme les sources privilégiées de connaissances sur la santé et les relations sexuelles chez les jeunes répondants. On a également fait remarquer que la plupart des ménages du Nunavut n'ont pas accès à Internet et n'utilisent que des navigateurs publics qui peuvent bloquer par inadvertance du contenu sur la santé sexuelle en ayant I'intention de bloquer du contenu pornographique (18). De plus, les déterminants sociaux de la santé, y compris les logements inadéquats, surpeuplés et les taux de chômage élevés, sont étroitement liés à la consommation de substances dans le territoire (21). Les substances les plus souvent consommées sont l'alcool et la marijuana, et la consommation excessive d'alcool est courante. II y a peu de données canadiennes disponibles à 
ce sujet, toutefois les personnes qui consomment des drogues sont plus susceptibles de signaler de la stigmatisation et de la méfiance à l'égard du système de santé. Ces comportements peuvent contribuer à une diminution de l'utilisation des soins de santé et à la réticence à désigner et à localiser des partenaires sexuels (22).

Des représentants en santé communautaire et d'autres fournisseurs de soins de santé ont souligné l'importance du choix de la langue dans la prestation des services de santé au Nunavut. De nombreux programmes de promotion de la santé dans les programmes de santé publique sont élaborés par des personnes dont la langue maternelle est l'anglais. La majorité des résidents du Nunavut considèrent l'inuktitut comme leur langue maternelle. La structure linguistique, le vocabulaire, les systèmes sonores, la grammaire et d'autres considérations sont importants et influents sur le sens et la communication. C'est particulièrement vrai lorsqu'on fait référence à des concepts liés à la santé et à la santé sexuelle. Des ressources comme le projet « Tukisiviit : Do You Understand? » avec un glossaire sur la santé sexuelle ont été décrites comme très utiles (23).

Les petites collectivités présentent à la fois des avantages et des défis en matière de gestion de la syphilis. Les personnes infectées par la syphilis peuvent être préoccupées par la confidentialité de leurs renseignements personnels sur la santé dans les petites collectivités et, par conséquent, éviter de se rendre au centre de santé local où un membre de leur famille peut travailler. De plus, l'accès aux services médicaux dans la plupart des mines est limité, ce qui peut retarder le diagnostic et le traitement des cas et des contacts. Le fait que de nombreuses collectivités soient éloignées entraîne un très long délai entre le prélèvement de spécimens et la déclaration des résultats. Ce délai peut entraîner une transmission continue de l'infection ainsi qu'une progression de la maladie. L'une des façons d'atténuer ce retard est de recourir aux tests au point de service pour la détection de la syphilis. À l'heure actuelle, il n'y a pas de test de dépistage de la syphilis au point de service approuvé par Santé Canada. Une initiative financée par les Instituts de recherche en santé du Canada a été lancée au Nunavut et au Nunavik (une région qui comprend le tiers nord de la province de Québec) afin d'évaluer l'acceptabilité, le rendement et l'utilité d'un test double de la syphilis au point de service (24). Toutefois, ce travail a été retardé en raison de la pandémie de COVID-19 et de considérations liées à la dotation. II est également important d'améliorer l'éducation des personnes à risque de contracter l'infection. À la demande des représentants en santé communautaire, des travaux sont en cours pour élaborer des affiches éducatives qui comprennent des traductions en inuktitut de textes et des affiches visuelles de lésions syphilitiques. Nous prévoyons que cette intervention et d'autres interventions de ce genre favoriseront l'utilisation des services de prévention des infections transmissibles sexuellement dans les collectivités, car des initiatives semblables dirigées par des pairs ont été couronnées de succès dans d'autres contextes (25-27). L'utilité des interventions de santé mobiles, comme les rappels par message texte pour le dépistage, n'a pas été évaluée au Nunavut, mais il a été démontré que de telles initiatives augmentent le dépistage de la syphilis et du $\mathrm{VIH}$, relient les personnes aux services et entraînent un changement de comportement (28-32).

Des travaux continus sont nécessaires pour continuer d'améliorer l'engagement communautaire et de fournir des soins de santé et des services de santé publique d'une manière aussi conforme que possible aux objectifs de vérité et de réconciliation. Cela servira à intégrer plus d'information sur les représentants en santé communautaire et les approches culturellement adaptées aux soins de santé dans la documentation universitaire, et à mettre fin à l'éclosion de syphilis en cours. Il faut également améliorer l'information sur les expériences des personnes qui ont accès aux soins de santé et aux services de santé publique.

\section{Conclusion}

Malgré les défis logistiques liés à la gestion de la syphilis dans le territoire, les résultats globaux ont été positifs, et aucun cas congénital confirmé n'a été identifié. Nous attribuons cela à un effort coordonné de multiples partenaires, notamment les mesures clés prises par les ISP et les représentants en santé communautaire.

\section{Déclaration des auteurs}

A. S. - Conception du rapport, rédaction et révision du document

J. P. - Conception du rapport

K. K. - Analyse et interprétation des données

Tous les auteurs ont examiné les versions préliminaires et contribué à la révision de l'article.

Le contenu de l'article et les points de vue qui y sont exprimés n'engagent que les auteurs et ne correspondent pas nécessairement à ceux du gouvernement du Canada.

\section{Intérêts concurrents}

Aucun.

\section{Remerciements}

Nous tenons à souligner le rôle inestimable que jouent les fournisseurs de soins de santé qui donnent des soins de santé et des services de santé publique aux Nunavummiuts, tant sur le territoire qu'ailleurs au Canada.

\section{Financement}

Aucun. 


\section{Références}

1. Nunavut Tunngavik Incorporated. Annual report on the state of Inuit culture and society, 2007-8. Iqaluit (NU): NTI; 2008 (accédé 2021-08-30). https://www.tunngavik.com/ publications/annual-report-on-the-state-of-inuit-culture-andsociety-2007-2008/

2. Inuit Tapiriit Kanatami. Comprehensive report on the social determinants of Inuit Health. Ottawa (ON): ITK; 2014 (accédé 2021-08-21). https://www.itk.ca/social-determinantscomprehensive-report/

3. Government of Nunavut. Nunavut Communicable Disease and Surveillance Manual. Section 6.4.1 Syphilis. Iqaluit (NU): Government of Nunavut; 2018 (accédé 2021-08-21). https:// www.gov.nu.ca/documents/communicable-disease-manual

4. Statistique Canada. Estimations de la population au $1^{\text {er }}$ juillet, par âge et sexe (accédé 2022-01-26). https:// www150.statcan.gc.ca/t1/tb/1/fr/tv.action?pid=1710000501 \&request_locale $=\mathrm{fr}$

5. Cherba M, Healey Akearok GK, MacDonald WA. Addressing provider turnover to improve health outcomes in Nunavut. CMAJ 2019;191(13):E361-4. DOI PubMed

6. Canadian Health Workforce Network. Comparative Review of Community Health Representatives Scope of Practice in International Indigenous Communities. NIICHRO; 2004. https://www.hhr-rhs.ca/index.php?option=com_ mtree\&task=viewlink\&link_id=7763\&lang=en

7. Pagaoa M, Grey J, Torrone E, Kreisel K, Stenger M, Weinstock $\mathrm{H}$. Trends in nationally notifiable sexually transmitted disease case reports during the US COVID-19 pandemic, January-December 2020. Sex Transm Dis 2021;48(10):798-804. DOI PubMed

8. Rac MW, Revell PA, Eppes CS. Syphilis during pregnancy: a preventable threat to maternal-fetal health. Am J Obstet Gynecol 2017;216(4):352-63. DOI PubMed

9. Poliquin V, Dhaliwal A, Lopez A, Bullard J. Local rate of Jarisch-Herxheimer reaction following penicillin treatment for syphilis during pregnancy. J Obstet Gynaecol Can 2020;42(5):689. DOI

10. Macumber $S$, Singh AE, Robinson J, Smyczek $P$, Sklar C, Gratix J, Rathjen L. Incidence and Outcomes of Jarisch-Herxheimer reactions following treatment for infectious syphilis in late pregnancy in Alberta Canada, 2015-2020. STI \& HIV World Congress. 2021;97 (Suppl 1):P438. https://sti.bmj.com/content/97/Suppl_1/ A173.1.abstract

11. Young TK, Tabish T, Young SK, Healey G. Patient transportation in Canada's northern territories: patterns, costs and providers' perspectives. Rural Remote Health 2019;19(2):5113. DOl PubMed

12. Hawkes SJ, Gomez GB, Broutet N. Early antenatal care: does it make a difference to outcomes of pregnancy associated with syphilis? A systematic review and meta-analysis.

PLoS One 2013;8(2):e56713. DOI PubMed
13. Zhang $X H, X u$ J, Chen DQ, Guo LF, Qiu LQ. Effectiveness of treatment to improve pregnancy outcomes among women with syphilis in Zhejiang Province, China. Sex Transm Infect 2016;92(7):537-41. DOI PubMed

14. Royal Commission on Aboriginal Peoples. Volume 3: Gathering Strength. Ottawa (ON): Canada Communications Group; 1996. https://data2.archives.ca/e/e448/e01118823003.pdf

15. He J, Wang Y, Du Z, Liao J, He N, Hao Y. Peer education for HIV prevention among high-risk groups: a systematic review and meta-analysis. BMC Infect Dis 2020;20(1):338. DOI PubMed

16. Dutcher MV, Phicil SN, Goldenkranz SB, Rajabiun S, Franks J, Loscher BS, Mabachi NM. "Positive Examples": a bottom-up approach to identifying best practices in HIV care and treatment based on the experiences of peer educators. AIDS Patient Care STDS 2011;25(7):403-11. DOI PubMed

17. Pauktuutit. Violence and Abuse Prevention: Residential Schools. Ottawa (ON): Pauktuutit (accédé 2021-08-15). https://www.pauktuutit.ca/abuse-prevention/children-andyouth/

18. Healey G. Youth perspectives on sexually transmitted infections and sexual health in Northern Canada and implications for public health practice. Int J Circumpolar Health 2016;75:1:30706. DOI

19. Healey GK, Meadows LM. Inuit women's health in Nunavut, Canada: a review of the literature. Int J Circumpolar Health 2007;66(3):199-214. DOI PubMed

20. Pauktuutit. Violence and Abuse Prevention: Children and Youth. Ottawa (ON): Pauktuutit (accédé 2021-09-01). https:// www.pauktuutit.ca/abuse-prevention/children-and-youth/

21. Canada Drug Rehab. Nunavut Overview \& Quick Facts. Canada Drug Rehab (accédé 2021-08-21). https://www. canadadrugrehab.ca/nunavut/nunavut-overview-quick-facts/

22. Kidd SE, Grey JA, Torrone EA, Weinstock HS. Increased methamphetamine, injection drug and heroin use among women and heterosexual men with primary and secondary syphilis - United States, 2013-2017. MMWR Morb Mortal Wkly Rep 2019;68(6):144-8. DOI PubMed

23. Pauktuutit. Tukisiviit-Glossary. Ottawa (ON): Pauktuutit; 2014 (accédé 2021-09-01). https://www.pauktuutit.ca/health/ sexual-health/tukisiviit-glossary/

24. Canadian Institutes of Health Research. Stopping Syphilis Transmission in Arctic Communities through Rapid Diagnostic Testing (STAR study) (accédé 2021-08-27). https://webapps.cihr-irsc.gc.ca/decisions/p/project_details. html?applld=388947\&lang=en

25. Conserve DF, Jennings L, Aguiar C, Shin G, Handler L, Maman S. Systematic review of mobile health behavioural interventions to improve uptake of HIV testing for vulnerable and key populations. J Telemed Telecare 2017;23(2):347-59. DOI PubMed 
26. Burns K, Keating P, Free C. A systematic review of randomised control trials of sexual health interventions delivered by mobile technologies. BMC Public Health 2016;16(1):778. DOI PubMed

27. Beattie TS, Mohan HL, Bhattacharjee $P$, Chandrashekar S, Isac S, Wheeler T, Prakash R, Ramesh BM, Blanchard JF, Heise L, Vickerman P, Moses S, Watts C. Community mobilization and empowerment of female sex workers in Karnataka State, South India: associations with HIV and sexually transmitted infection risk. Am J Public Health 2014;104(8):1516-25. DOI PubMed

28. Paschen-Wolff MM, Restar A, Gandhi AD, Serafino $S$, Sandfort T. A systematic review of interventions that promote frequent HIV testing. AIDS Behav 2019;23(4):860-74. DOI PubMed

29. Santos VD, Costa AK, Lima IC, Alexandre HO, Gir E, Galvão MT. Use of the telephone for accessing people living with HIV/AIDS to antiretroviral therapy: systematic review. Cien Saude Colet 2019;24(9):3407-16. DOI PubMed
30. Geldof M, Thiombiano BA, Wagner N. "When I receive the message, it is a sign of love": symbolic connotations of SMS messages for people living with HIV in Burkina Faso. AIDS Care 2020;33(6):810-17. DOI PubMed

31. Wirsiy FS, Nsagha DS, Njajou OT, Besong JB. A protocol for a randomized controlled trial on mobile phone text messaging to improve sexo-reproductive health in Cameroon. J Public Health Epidemiol 2020;12(3):179-85. $\mathrm{DOI}$

32. Muhindo R, Mujugira A, Castelnuovo B, Sewankambo NK, Parkes-Ratanshi R, Kiguli J, Tumwesigye NM, Nakku-Joloba E. Text message reminders and peer education increase HIV and Syphilis testing among female sex workers: a pilot quasi-experimental study in Uganda. BMC Health Serv Res 2021;21(1):436. DOI PubMed

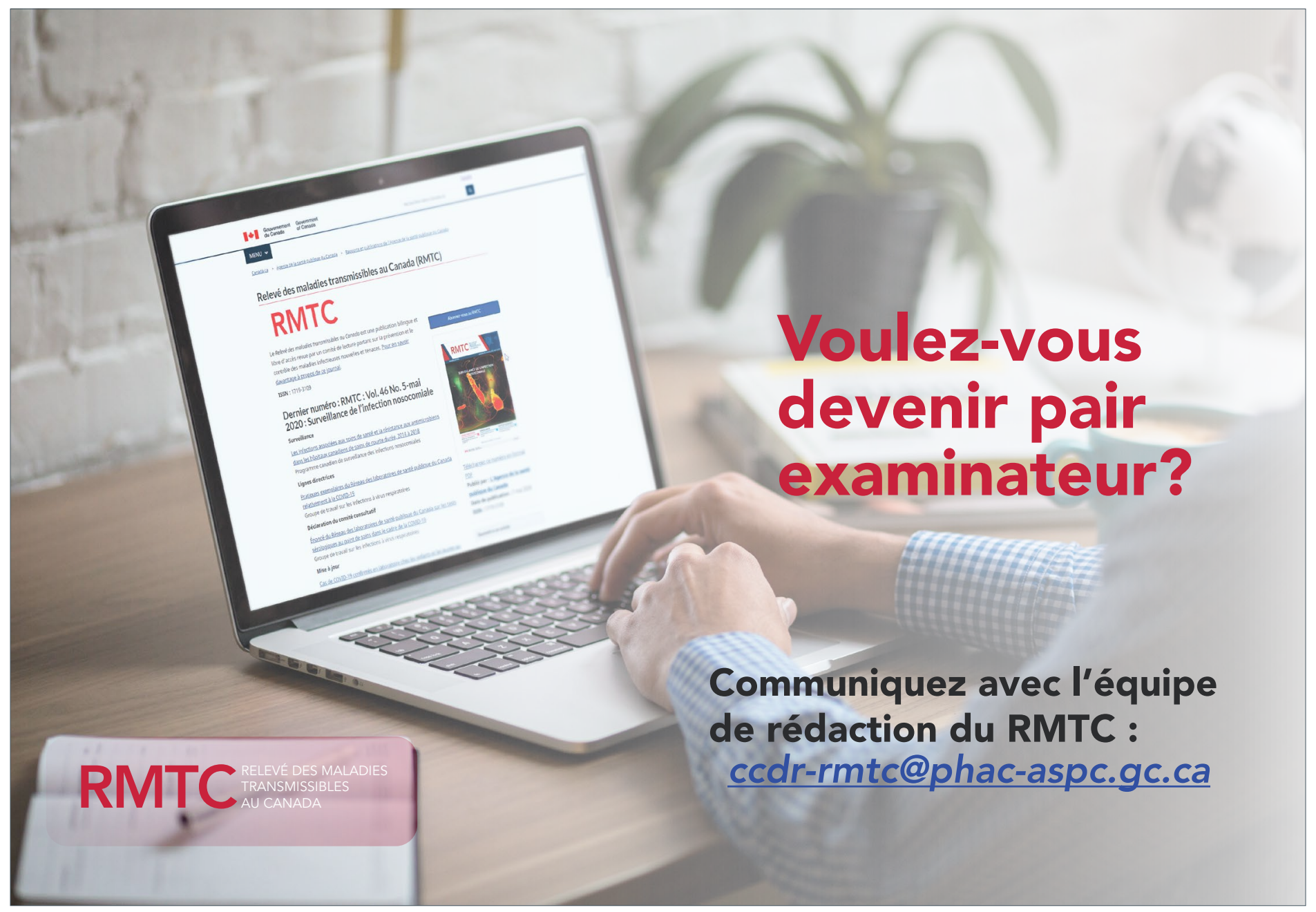

